\title{
Positive Effects of the Ultrasound on Biosynthesis, Characteristics and Antibacterial Activity of Silver Nanoparticles Using Fortunella Japonica
}

\author{
Nguyen P. Anh ${ }^{1,2}$, Duong N. Linh ${ }^{3}$, Nguyen V. Minh ${ }^{3}$ and Nguyen Tri1,* \\ ${ }^{1}$ Institute of Chemical Technology, 01 Mac Dinh Chi Str., HCM City, 70100, Vietnam \\ ${ }^{2}$ Graduate University of Science and Technology - VAST, 18 Hoang Quoc Viet Str., Hanoi, 10072, Vietnam \\ ${ }^{3}$ Faculty of Biotechnology, Ho Chi Minh City Open University, 97 Vo Van Tan Str., HCM City, 70100, Vietnam
}

Silver nanoparticles (AgNPs) were biosynthesized using Fortunella Japonica extract as an reducing agent through a rapidly and ecofriendly assisted ultrasound method. Effect of ultrasound assistant on synthesis duration, properties and antibacterial activity of AgNPs against Escherichia coli, Bacillus subtilis and Bacillus cereus were reported. The suitable duration for AgNPs assisted ultrasound biosynthesis was 90 minutes that is 60 minutes shorter than stirring-assisted method. The obtained AgNPs with a face centred cubic structure were nearly spherical in shape and uniform in size distribution with the average nanosize of $11.6 \mathrm{~nm}$, being smaller than the size of AgNPs prepared by stirring-assisted method. AgNPs sample exhibited effective antibacterial activity against all three bacteria with average diameter of inhibition zones of over $15 \mathrm{~mm}$ and minimum inhibitory concentration of $4.13 \mu \mathrm{g} / \mathrm{mL}$. The results showed that the ultrasound assistant has positive effects such as shortening synthesis duration, reducing average particle size and increasing antibacterial activity of AgNPs.

[doi:10.2320/matertrans.M2019065]

(Received March 5, 2019; Accepted June 26, 2019; Published August 25, 2019)

Keywords: ultrasound assistant, silver nanoparticles, antibacterial activity, Fortunella Japonica

\section{Introduction}

Bacterial resistance to antibiotics has become one of the major concerns in both public health and worldwide environment. 1) Bacteria may develop tolerance toward antibiotics through mutations in their own genome or by acquisition of resistance-conferring genes via horizontal gene transfer, with the latter being considered as a more prominent mechanism. ${ }^{2,3)}$ Resistance to antibiotics in either pathogenic, commensal, or environmental bacteria has led to an increase in treatment expenses, higher mortality rates and less clinical efficacy. So, development of new antimicrobial agent becomes a challenging task for researchers. The use of nanoparticles (NPs) was known as an effective solution to against antibiotic-resistant strains. ${ }^{4)}$ Among NPs, silver nanoparticles (AgNPs) are one of the most extensively used materials, largely due to effective antimicrobial properties. ${ }^{5)}$ The potent antibacterial properties of AgNPs may be attributed to released $\mathrm{Ag}$ ions, which could have interaction with microorganisms by means of their attaching to the surface of the cell membranes of bacteria and penetrating into the bacterial cells. In the bacterial cells, AgNPs could interact with sulfur- and phosphorus-containing compounds like DNA of bacterial to give rise to the deadly impairment of them. ${ }^{6}$ )

Friendly synthetic processes of AgNPs using non-toxic chemicals such as microorganisms, ${ }^{7)}$ plant extracts, ${ }^{8)}$ and natural polymers ${ }^{9)}$ as reducing agents have emerged as simple, cost-effective and eco-friendly alternatives for AgNPs synthesis. The exploit of green chemistry for AgNPs synthesis has obtained substantial achievements in the latest years. Literature review shows that AgNPs biosynthesis focused on several plant and microbial sources, in which plant parts like seeds, ${ }^{10}$ leaf, ${ }^{11)}$ bark, ${ }^{12)}$ stem, ${ }^{13)}$ and fruit extracts $^{14)}$ have been effectively used. The association of

*Corresponding author, E-mail: ntri@ict.vast.vn nanotechnology and green chemistry has unfolded the methods of AgNPs synthesis. In the previous studies. ${ }^{15,16)}$ AgNPs were synthesized from aqueous silver nitrate solution using Lemon Citrus Latifolia (LCL) and Fortunella Japonica $(F J)$ extracts by stirring-assisted method. The reduction accomplished principally due to the combination of different components ( $L C L, F J$ extracts) and sunlight illumination played a vital role in AgNPs formation. This fact demonstrates that the use of $L C L$ and $F J$ extracts as combined reducing and stabilizing agent is two of the most efficient and promising methods in the AgNPs preparation. AgNPs solutions exhibited an effective activity against bacteria, i.e. Escherichia coli (E. coli), Bacillus cereus (B. cereus), and Bacillus subtilis (B. subtilis). However, the minimum inhibitory concentration on these bacteria of obtained AgNPs had not been reported so far.

Prathna et al. ${ }^{17)}$ indicated that citric acid in the lemon juice plays a main role as a reducing agent in the reduction of $\mathrm{Ag}^{+}$ ions to AgNP. The reaction mechanism for the formation of AgNPs using lemon juice as reducing agent can be written as follows:

$$
\begin{aligned}
& 4 \mathrm{Ag}^{+}+\mathrm{C}_{6} \mathrm{H}_{5} \mathrm{O}_{7}+\mathrm{X}+2 \mathrm{H}_{2} \mathrm{O} \\
& \quad \rightarrow 4 \mathrm{Ag}^{0}+\mathrm{C}_{6} \mathrm{H}_{5} \mathrm{O}_{7} \mathrm{H}_{3}+\mathrm{H}^{+}+\mathrm{Y}+\mathrm{O}_{2} \uparrow
\end{aligned}
$$

where $\mathrm{X}$ is the other bio-organic substances present in lemon extract which along with citric acid helped in reduction of $\mathrm{Ag}^{+}$ions. After the reaction process, $\mathrm{X}$ on reducing $\mathrm{Ag}^{+}$ions gets itself oxidized to products $\mathrm{Y}$.

Currently, ultrasound assisted manner for preparing nanoparticles is considered as an eco-friendly and green approach. ${ }^{18)}$ It is well known that ultrasound radiation in liquids has a variety of physical and chemical effects, which can provide a unique way of driving chemical reactions under extreme conditions. ${ }^{19)}$ The reduction of metal ions by an ultrasound radiation method has been used in a smaller extent to prepare surfactant or polymer-stabilised metal NPs, such as silver, gold and platinum. ${ }^{19-21)}$ Enhanced productivity was 
achieved by the application of ultrasound into processes. However, less papers on ultrasound assisted biosynthesis of AgNPs have been reported.

With the aim to propose an effective ecofriendly method with shorter synthesis duration and more uniform in AgNPs size distribution, the combination of ultrasound and sunlight illumination was employed for biosynthesis of AgNPs using $F J$ extract in this study. The effect of ultrasound on the synthesis duration, nanosize and bactericidal activity of AgNPs was also investigated. The antibacterial performances of AgNPs including diameter of the inhibition zone and minimum inhibitory concentration were evaluated with three bacteria - E. coli, B. subtilis and B. cereus.

\section{Experimental}

FJ extracts and the solution of AgNPs were prepared base on our previous work. ${ }^{15)}$ Silver nitrate $\left(\mathrm{AgNO}_{3}\right.$, Merck, $>99.8 \%$ ) purchased from Merck was used as a precursor for AgNPs synthesis. To test the effect of ultrasonic homogenization method on AgNPs synthesis, the experiments were conducted at the suitable conditions, determined in our previous work. ${ }^{15)}$ FJ extract was mixed with $\mathrm{AgNO}_{3}$ $1.75 \mathrm{mM}$ solution with the volume ratio of $3.5 \mathrm{AgNO}_{3}$ solution/1.5 F.J. Extract, stirred $300 \mathrm{rpm}$ for 150 minutes at $40^{\circ} \mathrm{C}$ under sunlight illumination. In this work, stirring was replaced by ultrasonic and synthesis duration was redetermined. The sample was put in the ultrasonic tank (model $\mathrm{LC} 30 \mathrm{H}$, Germany) with the frequency of $35 \mathrm{kHz}$. The silver nanoparticle solution was purified by thrice centrifugation in distilled water at $5000 \mathrm{rpm}$ in 30 minutes for each time. The captured solid mixture was dried at $60^{\circ} \mathrm{C}$ for 24 hours to obtain AgNPs powder.

The presence of AgNPs in the solution was determined by UV-Vis spectrophotometer (UV-1800, Shimadzu). The samples were diluted 5 times before being analyzed. The recorded spectra were then replotted using Origin software.

The characteristics of AgNPs samples were determined by the techniques detailed in our previous works. ${ }^{15,16)}$

The obtained AgNPs samples have been tested for antibacterial activity against $E$. coli ATCC 25922, B. subtilis ATCC 6633 and B. cereus ATCC 14579 using an agar well diffusion method. ${ }^{16)}$ The results were read by measuring the diameter of inhibition zone $(\mathrm{mm})$ around the agar. The experiments were repeated for three times to attain an average value.

To examine the minimum inhibitory concentration of AgNPs against three bacteria, the different concentrations of AgNPs (N/2, N/4, N/8, N/16, N/32, N/64, and N/128 with $\mathrm{N}$ was the initial concentration of AgNPs solution in deionized water, $\mathrm{N}=132.3 \mu \mathrm{g} / \mathrm{mL}$ ) were prepared by diluting AgNPs solution with deionized water. Subsequently, the diluted samples were mixed with the sterile nutrient agar. By using sterile sticks, the standardized inoculum of each selected bacteria with $1.5 \times 10^{\wedge} 7 \mathrm{CFU} / \mathrm{ml}$ were inoculated on agar plates mixed with AgNPs samples from low to high concentrations. A plate of the sterile nutrient agar was not mixed with AgNPs for control. ${ }^{22)}$ Each strain of bacteria was inoculated at a point on a disk with the same location on the disks. Finally, the plates were incubated at $37^{\circ} \mathrm{C}$ for 24 hours.

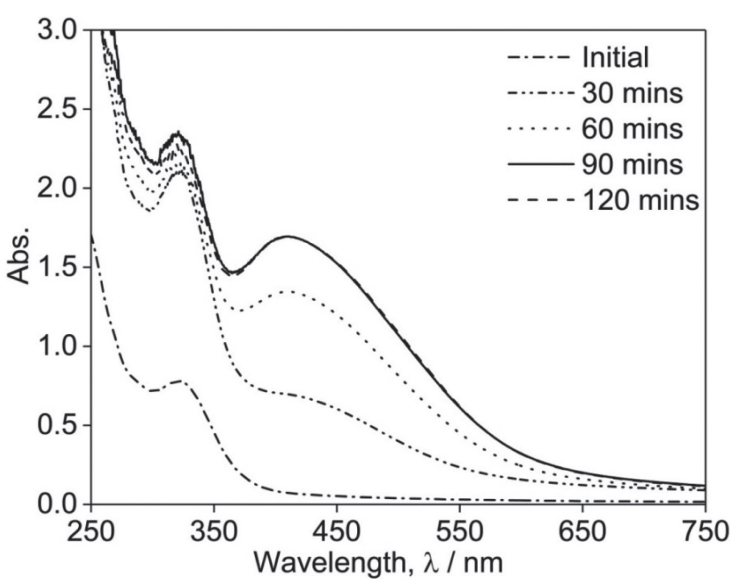

Fig. 1 UV-Vis spectral analysis of obtained AgNPs suspension samples from FJ extract assisted ultrasound.

The lowest concentration of AgNPs that inhibits the growth of tested bacteria was considered as the minimum inhibitory concentration (MIC). ${ }^{23)}$

\section{Results and Discussions}

\subsection{Effect of ultrasonic assistant on the synthesis duration and characteristics of AgNPs}

The band indicating AgNPs in the solution with the wavelength range of 400-450 nm (Fig. 1) shows that AgNPs were effectively synthesized by applying ultrasonication. The synergistic effect of ultrasonic cavitation and extracts was responsible for immediate formation of AgNPs. With ultrasonic assistant, the complete conversion of $\mathrm{Ag}^{+}$to $\mathrm{Ag}^{0}$ occurred within 90 minutes which was evident from the absorbance values. Further increase in synthesis duration did not exhibit any significant increase in absorption value. Compared with AgNPs synthesized by stirring-assisted method with the optimal synthesis duration of 150 minutes, ${ }^{15}$ ) this duration was 60 minutes shorter. This seems to be an interesting approach towards the more rapidly and ecofriendly AgNPs synthesis.

X-ray diffraction analysis (XRD) result of AgNPs synthesized with assisted ultrasound from $F J$ extract was shown in Fig. 2. The diffraction position at $2 \theta=38.5,46.7$, 64.5 and $77.3^{\circ}$ corresponding to (111), (200), (220) and (311) reflection planes corresponded to AgNPs structure. In addition, it could be asserted that the (111) crystallographic plane was more predominant than other planes. The presence of higher atom density could be the reason to enhance the grain growth along (111) orientation. ${ }^{24)}$ Beside, a few intense and unassigned peaks at $2 \theta=28.1,32.4,55.6$, and $57.0^{\circ}$ in the vicinage of silver peaks exhibited the presence of bioorganic phase crystals, ${ }^{25}$ ) which were present in $F J$ extract and responsible for silver ions reduction and stabilization of nanoparticles. The intensity of Bragg reflections on the sample suggested strong X-ray scattering centers in the crystalline phase and could possibly arise from the compounds in the nanoparticles during AgNPs synthesis. XRD pattern obtained was similarly to that of AgNPs synthesized with stirring-assisted way. ${ }^{15)}$ Based on the XRD result, the average crystal size of the sample at the (111) 


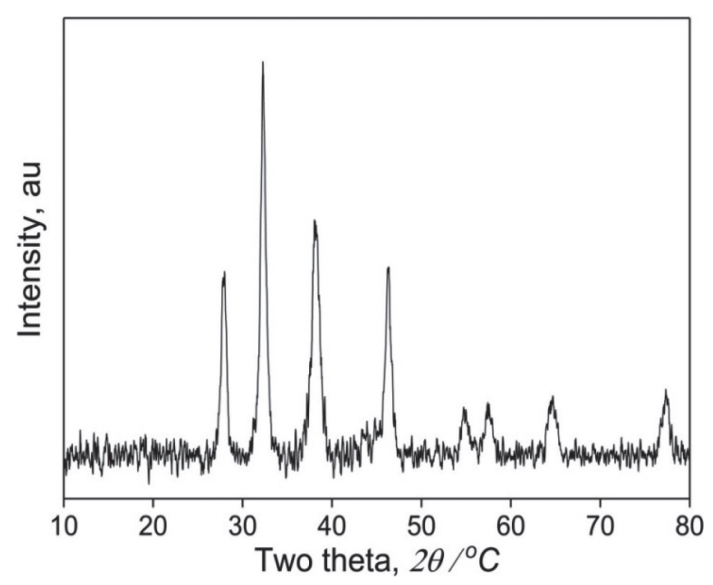

Fig. 2 XRD diffraction pattern of synthesized AgNPs with the assisted ultrasound after dried at $60^{\circ} \mathrm{C}$ for 24 hours.

crystallographic plane was determined following Scherrer's equation with $\mathrm{K}=0.94$. The average size of AgNPs synthesized in this work reached $11.2 \mathrm{~nm}$, smaller than that of AgNPs synthesized by stirring-assisted $\left.(\mathrm{d}=15.9 \mathrm{~nm}) .{ }^{15}\right)$ FT-IR spectra of the sample synthesized by assisted ultrasound (Fig. 3) also appeared typical bands of silver nanoparticles, similar to the results obtained of AgNPs from FJ extract by stirring-assisted method in our previous study. ${ }^{15)}$

The result of transmission electron microscopy (TEM) analysis of AgNPs sample (Fig. 4(a)) indicated that nanoparticles were polydispersed and spherical in shape. The image clearly showed a transparent organic layer coating around AgNPs. This layer was due to surrounded phytochemicals in the extracts that served as a capping agent to prevent agglomeration. The inter-particle separation among nanoparticles confirmed the presence of organic molecules like polyphenols capping on AgNPs. From TEM image, by using the ImageJ software, the size distribution of AgNPs sample was elucidated in Fig. 4(b), and the average size of obtained AgNPs was determined of approximately $11.6 \mathrm{~nm}$. This result was consistent with
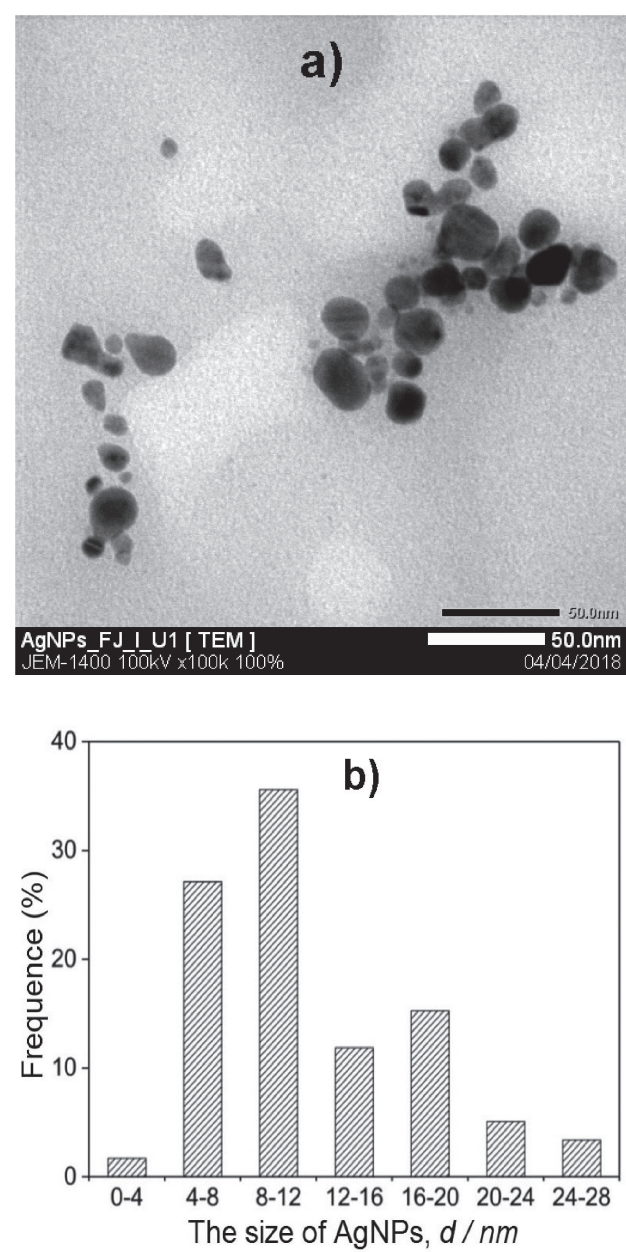

Fig. 4 TEM image (a) and the size distribution histogram (b) of AgNPs synthesized using FJ extract with assisted ultrasound.

XRD pattern. The average size of AgNPs formed with assisted ultrasound was much lower than those obtained by stirring-assisted approach from the same extract $(15.9 \mathrm{~nm}){ }^{15)}$ Hence, it was clear that application of ultrasonic assistant had reduced significantly both the synthesis duration and the average size of AgNPs.

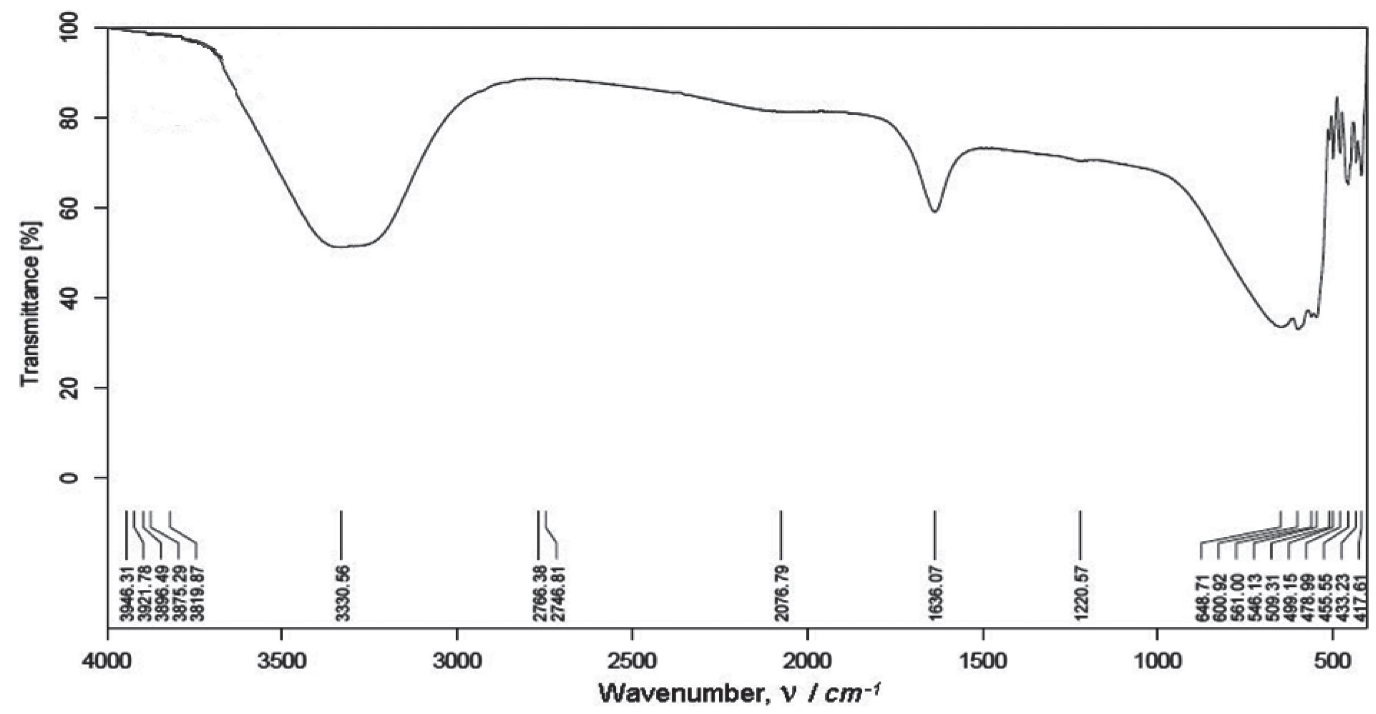

Fig. 3 FT-IR spectra of AgNPs solution. 


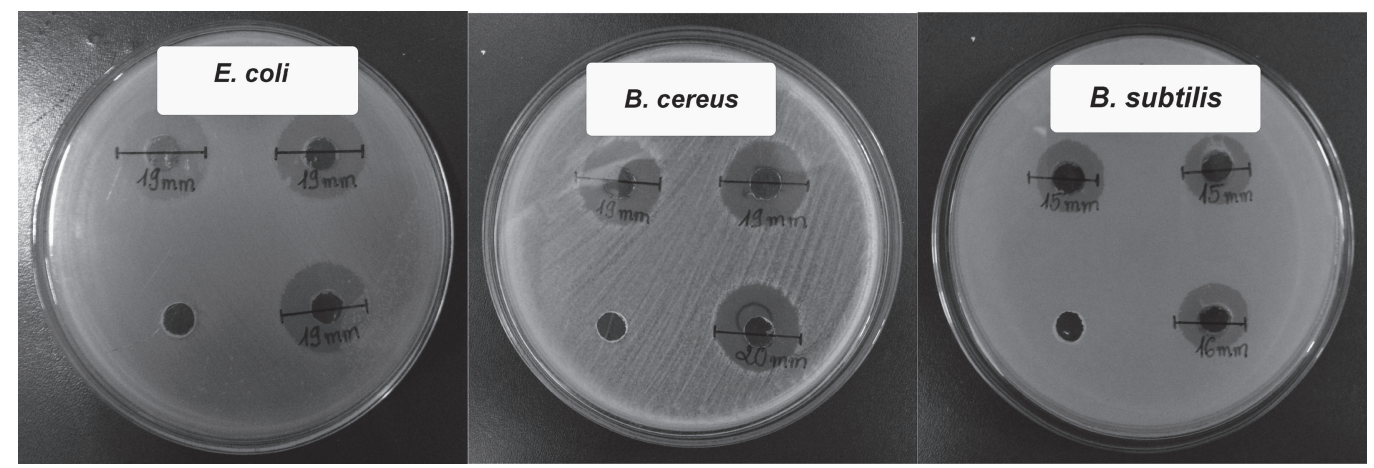

Fig. 5 Images of inhibition zone against E. coli, B. cereus, and B. subtilis of AgNPs sample synthesized by ultrasound assisting.
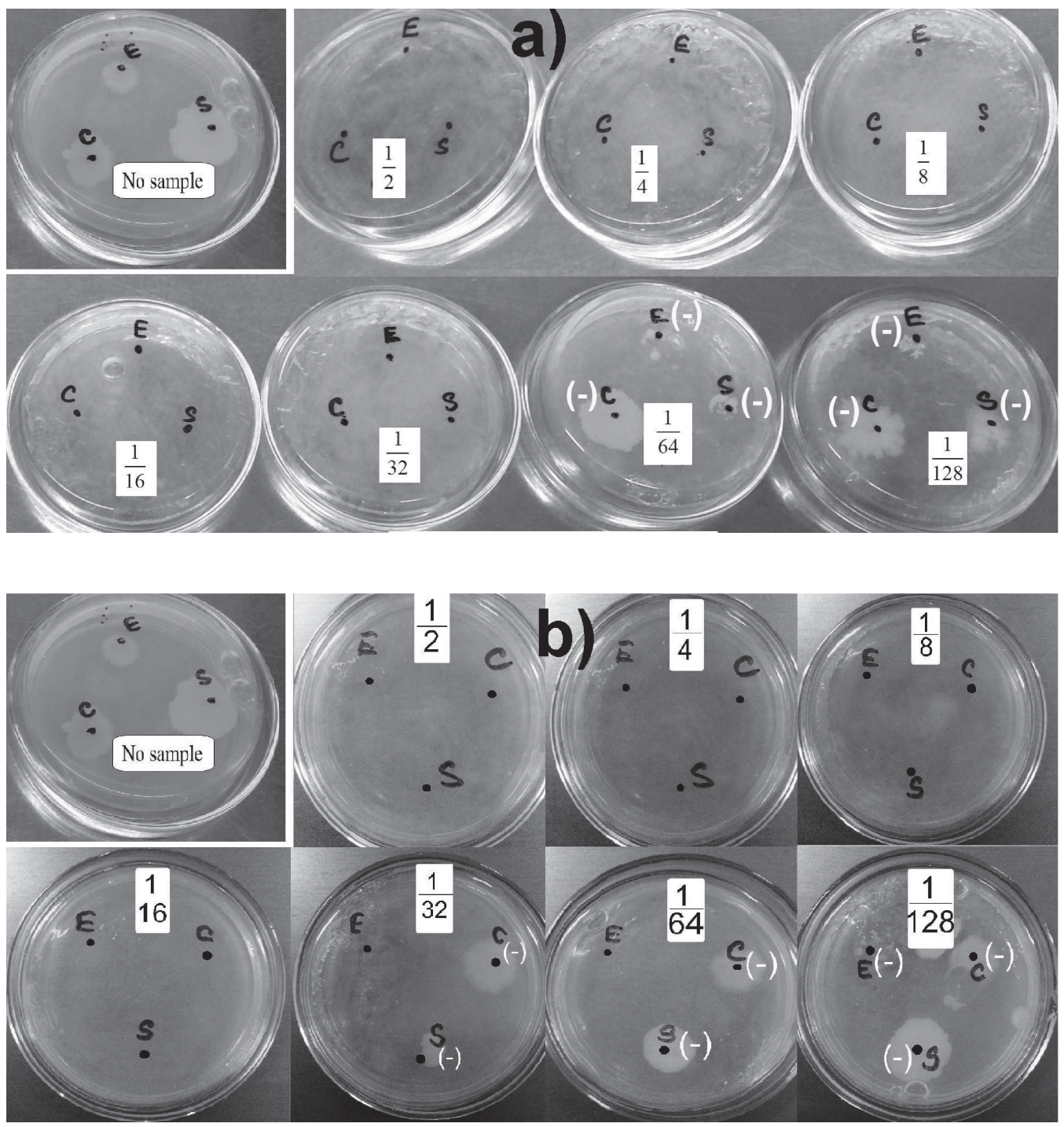

\section{(-): Bacteria colonies were detected.}

Fig. 6 The comparison of the minimum inhibitory concentrations of two AgNPs samples synthesized with assisted ultrasound (a - AgNPs-U) and stirring (b - AgNPs-S) against E. coli (E), B. cereus (C), and B. subtilis (S).

\subsection{Effect of ultrasonic assistant on the antibacterial} activity of AgNPs

Figure 5 showed that AgNPs synthesized by ultrasound assistant way performed efficient antibacterial activity against all three bacteria $-E$. coli, B. subtilis and B. cereus. The zones of clearance against E. coli, B. cereus and B. subtilis were determined $18.0,19.3$ and $15.3 \mathrm{~mm}$, respectively. The antibacterial activity was almost closed to the ability of AgNPs formed by stirring method in previous report. ${ }^{15)}$ This fact demonstrated that assisted ultrasound biosynthesis did 
not affect considerably the antibacterial activity of AgNPs product. The nanosize range of AgNPs obtained by these two homogenization methods was almost equal. The average inhibition zone diameter against $E$. coli of AgNPs in this work was higher than that of other studies such as Pomegranate fruit seeds, ${ }^{26}$ Nicotiana tobaccum leaf, ${ }^{27)}$ Lemon extract, ${ }^{28)}$ and Neem leaves. ${ }^{29)}$ This could be explained that the much smaller size of AgNPs exhibited higher antibacterial due to higher surface area and faster release of silver ions.

The antibacterial activities of AgNPs samples were further determined by their corresponding minimum inhibitory concentration (MIC). Figure 6 presented the inhibition zone against E. coli, B. cereus and B. subtilis treated with AgNPs solutions under different concentrations of two AgNPs samples synthesized by ultrasound (Fig. 6(a)) and stirring (Fig. 6(b)). It was observed that the exponential phase of bacteria delayed in the presence of AgNPs and this phenomenon was more obvious with the increase of AgNPs concentration. AgNPs sample assisted the ultrasound could delay the exponential phase of all bacteria E. coli, B. cereus and $B$. subtilis and could completely inhibit the bacterial growth at the similar MIC of $4.13 \mu \mathrm{g} / \mathrm{mL}(\mathrm{N} / 32)$. The MIC of AgNPs synthesized with stirring-assisted method against E. coli, B. cereus and B. subtilis reached 4.13, 8.27, 8.27 $\mu \mathrm{g} / \mathrm{mL}$, respectively. Thus, it showed that AgNPs assisted ultrasound biosynthesis led to a reduction of the MIC against gram (+) bacteria - B. cereus and B. Subtilis (shown in Fig. 7).

The mechanism of antibacterial activity of silver nanoparticles was partially known, it reported that the bactericidal activity of AgNPs was due to its the negatively charged bacterial cell wall membrane and electrostatic interaction between positively charged nanoparticles. ${ }^{30)}$ It also has been reported that smaller size of AgNPs are known to exhibit higher toxicity because of higher surface area and faster release of AgNPs species. ${ }^{31)}$ Compared to stirring-assisted synthesis, the smaller nanoparticles obtained from the assisted ultrasound method could easily reach the nuclear content of bacteria and have the larger surface, leading to an increase in the antibacterial activity of AgNPs. These results demonstrated the size-dependent antibacterial activities of AgNPs.

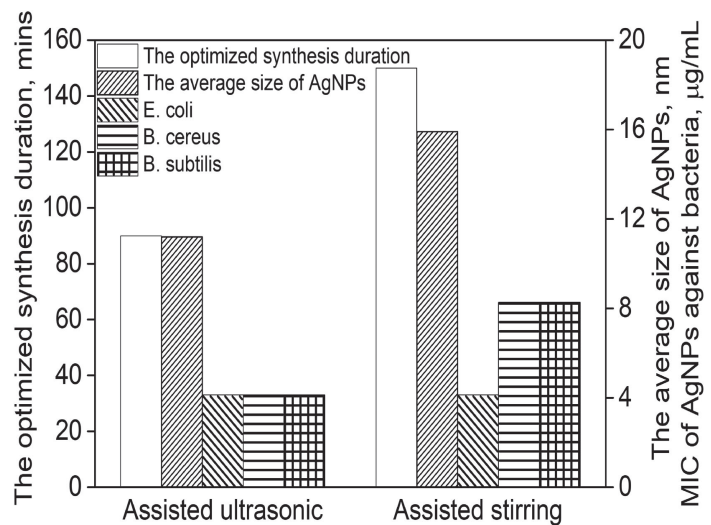

Fig. 7 The comparison of the optimized synthesis duration, average size, and MIC against bacteria of AgNPs prepared by assisting ultrasound and stirring using the same FJ extract.
To sum up, the implication of assisting ultrasound in AgNPs biosynthesis has reduced significantly the process duration, the average nanosize and the minimum inhibitory concentration of formed products (illustrated in Fig. 7).

\section{Conclusion}

The ultrasound assistant in biosynthesis has reduced significantly the process duration, average nanosize and minimum inhibitory concentration against bacteria of obtained AgNPs sample. Synthesized AgNPs were formed as highly crystallized spherical particles with the average size of $11.6 \mathrm{~nm}$. By ultrasound assistant method, AgNPs obtained were smaller and more uniform than the ones synthesized by assisted stirring way. Furthermore, the former AgNPs showed the efficient antibacterial activity against both gram (+) and gram (-) bacteria. Hence, this work has proposed a rapidly and eco-friendly method for synthesizing AgNPs with the small nanosize and highly effectiveness against human pathogenic bacteria. This method is also applicable in various fields, i.e. medical devices and antibacterial systems.

\section{REFERENCES}

1) R.J. Fair and Y. Tor: Perspect. Med. Chem. 6 (2014) PMC.S14459.

2) C.J. von Wintersdorff, J. Penders, J.M. van Niekerk, N.D. Mills, S. Majumder, L.B. van Alphen, P.H. Savelkoul and P.F. Wolffs: Front. Microbiol. 7 (2016) 1-10.

3) A.H. Holmes, L.S. Moore, A. Sundsfjord, M. Steinbakk, S. Regmi, A. Karkey, P.J. Guerin and L.J. Piddock: Lancet 387 (2016) 176-187.

4) R. Singh, M. Smitha and S.P. Singh: J. Nanosci. Nanotechnol. 14 (2014) 4745-4756.

5) A. Panáček, L. Kvítek, M. Smékalová, R. Večeřová, M. Koláŕ, M. Röderová, F. Dyčka, M. Šebela, R. Prucek and O. Tomanec: Nat. Nanotechnol. 13 (2018) 65-71.

6) D. Wu, W. Fan, A. Kishen, J.L. Gutmann and B. Fan: J. Endod. 40 (2014) 285-290.

7) V.K. Sharma, R.A. Yngard and Y. Lin: Adv. Colloid Interface Sci. 145 (2009) 83-96.

8) J.Y. Song and B.S. Kim: Bioprocess Biosyst. Eng. 32 (2009) 79-84.

9) H. Huang and X. Yang: Carbohydr. Res. 339 (2004) 2627-2631.

10) H. Bar, D.K. Bhui, G.P. Sahoo, P. Sarkar, S. Pyne and A. Misra: Colloids Surf. A 348 (2009) 212-216.

11) K.B. Narayanan and N. Sakthivel: Mater. Lett. 62 (2008) 4588-4590.

12) M. Sathishkumar, K. Sneha, S. Won, C.-W. Cho, S. Kim and Y.-S. Yun: Colloids Surf. B 73 (2009) 332-338.

13) P. Daisy and K. Saipriya: Int. J. Nanomedicine 7 (2012) 1189-1202.

14) B. Ankamwar, C. Damle, A. Ahmad and M. Sastry: J. Nanosci. Nanotechnol. 5 (2005) 1665-1671.

15) N.P. Anh, T.T.A. Mi, D.H.T. Linh, N.T.T. Van, H.T. Cuong, N.V. Minh and N. Tri: IACSIT Int. J. Eng. Technol. 7 (2018) 1570-1575.

16) D.H. Linh, N.P. Anh, T.T. Mi, N.T. Tinh, H.T. Cuong, T.L. Quynh, N.T. Van, N.V. Minh and N. Tri: Mater. Trans. 59 (2018) 1501-1505.

17) T. Prathna, N. Chandrasekaran, A.M. Raichur and A. Mukherjee: Colloids Surf. B 82 (2011) 152-159.

18) Z. Yuan, Z.-h. Chen, D. Chen and Z.-t. Kang: Ultrason. Sonochem. 22 (2015) 188-197.

19) X. Zhang and C. Xiao: J. Photochem. Photobiol. B 181 (2018) 122126.

20) P. Jawaid, M.U. Rehman, Q.-L. Zhao and T. Kondo: Free Radic. Biol. Med. 100 (2016) S124.

21) A. Ziylan-Yavaş and N.H. Ince: Chemosphere 162 (2016) 324-332.

22) P. Wayne: Clinical and Laboratory Standards Institute (CLSI) M100S23 (2013).

23) J. Washington and G. Wood: Manual of Clinical Microbiology, (ASM Press, Washington, 1995) pp. 1327-1331. 
24) I. Capek: Nanostructure Science and Technology, (Springer, Berlin, 2017) pp. 169-170.

25) A.A. AbdelHamid, M.A. Al-Ghobashy, M. Fawzy, M.B. Mohamed and M.M. Abdel-Mottaleb: ACS Sustainable Chem. Eng. 1 (2013) $1520-1529$.

26) S. Chauhan, M.K. Upadhyay, N. Rishi and S. Rishi: Int. J. Nanomater Biostruct. 1 (2011) 17-21.

27) K.S. Prasad, D. Pathak, A. Patel, P. Dalwadi, R. Prasad, P. Patel and K. Selvaraj: Afr. J. Biotechnol. 10 (2011) 8122-8130.

28) M.P. Link, A.M. Goorin, A.W. Miser, A.A. Green, C.B. Pratt, J.B
Belasco, J. Pritchard, J.S. Malpas, A.R. Baker and J.A. Kirkpatrick: N. Engl. J. Med. 314 (1986) 1600-1606.

29) A. Verma and M.S. Mehata: J. Radiat. Res. Appl. Sci. 9 (2016) 109115 .

30) P. Kumar, S. Senthamil Selvi, A. Lakshmi Prabha, K. Prem Kumar, R. Ganeshkumar and M. Govindaraju: Nano Biomed. Eng. 4 (2012) $12-16$

31) Z.-M. Xiu, Q.-B. Zhang, H.L. Puppala, V.L. Colvin and P.J. Alvarez: Nano Lett. 12 (2012) 4271-4275. 